This is the author's copy of the publication as archived with the DLR's electronic library at http://elib.dlr.de. Please consult the original publication for citation.

\title{
Electromyography for Teleoperated Tasks in Weightlessness
}

Hagengruber, Annette and Leipscher, Ulrike and Eskofier, Bjoern M and Vogel, Jörn

\section{Copyright Notice}

(C)2021 IEEE. Personal use of this material is permitted. However, permission to reprint/republish this material for advertising or promotional purposes or for creating new collective works for resale or redistribution to servers or lists, or to reuse any copyrighted component of this work in other works must be obtained from the IEEE.

\section{Citation Notice}

@ARTICLE hhagengruber2021electromyography,

title $=\{$ Electromyography for Teleoperated Tasks in Weightlessness $\}$

author $=\{$ Hagengruber, Annette and Leipscher, Ulrike and Eskofier, Bjoern M and Vogel, J $\{\backslash " 0\} r n\}$,

journal=\{51\} Trans,

volume $=\{51\}$,

pages $=\{130--140\}$

pages $=\{130--1$
year $=\{2021\}$,

publisher $=\{$ IEEE $\}$ 


\title{
Electromyography for teleoperated tasks in weightlessness
}

\author{
Annette Hagengruber, Ulrike Leipscher, Bjoern M. Eskofier, and Jörn Vogel
}

\begin{abstract}
The cooperation between robots and astronauts will become a core element of future space missions. This is accompanied by the demand for suitable input devices. An interface based on electromyography (EMG) represents a small, light and wearable device to generate a continuous 3D control signal from voluntarily muscle activation of the operator's arm. We analyzed the influence of microgravity on task performance during a 2D task on a screen. Six subjects performed aiming and tracking tasks in parabolic flights. Three different levels of fixation - fixed feet using foot straps, semi-free by using a foot rail, and free-floating feet - were tested to investigate how much user fixation is required to operate via the interface. The user study showed that weightlessness affects the usage of the interface only to a small extent. Success rates between $89 \%$ and $96 \%$ were reached within all conditions during microgravity. A significant effect between $0 G$ and $1 G$ could not be identified for the test series of fixed and semi-free feet, while free-floating feet showed significantly worse results in fine and gross motion times in $0 \mathrm{G}$ compared to ground tests (with success rates of $92 \%$ for $0 \mathrm{G}$ and 99\% for 1G). Further adaptation to the altered proprioception may be needed here. Hence, foot rails as already mounted in the ISS would be sufficient to use the interface in weightlessness. Low impact of microgravity, high success rates, and an easy handling of the system, indicates a high potential of an EMGbased interface for teleoperation in space.
\end{abstract}

Index Terms-Human Machine Interfaces, Electromyography, Space Application, Teleoperation, Robotcontrol, Weightlessness

\section{INTRODUCTION}

The interaction with remote controlled systems, in particular with robots, will become a core element of future space missions. Manned Space Explorations to the Moon and further to Mars are one of the main goals described in the Global Exploration Roadmap of the International Space Exploration Coordination Group (ISECG) [1]. The "Human-Robotic Partnership" is one of the mentioned principles to guide these developments, as the cooperation of human and robot can help to achieve the goals effectively, cost-efficiently, and safely for the astronauts.

The applications for such teleoperated robotic systems are manifold during space missions. They range from intravehicular activities (IVA) and extravehicular activities (EVA) to tasks in deep space or on planetary surface environments [2]. Different requirements of the remote task like location (EVA or IVA), complexity, or the level of precision demand various and appropriate input devices for astronauts.

A. Hagengruber, U. Leipscher, and J. Vogel are with the Institute of Robotics and Mechatronics, German Aerospace Center (DLR), Oberpfaffenhofen, Germany.

A. Hagengruber and B. M. Eskofier are with the Machine Learning and Data Analytics Lab, Department of Artificial Intelligence in Biomedical Engineering, Friedrich-Alexander University Erlangen-Nürnberg, Germany.

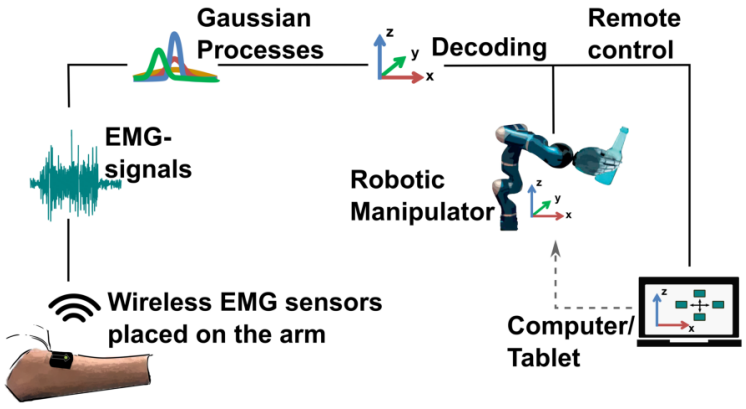

Fig. 1. Overview of the interface based on electromyography (EMG). Wireless, wearable sensors measure the muscular activity. A directional command is decoded with the help of Gaussian Process Regression. A possible application is the teleoperation of remote controlled systems like a robot.

In general, terrestrial applications for teleoperation offer a huge variety of input devices to cover different demands of the remote task. These range from $2 \mathrm{D}$ and $3 \mathrm{D}$ input devices, like tablets and conventional joysticks, over multi-D-input devices such as the sigma 7 of force dimension [3] or the DLR HUG [4]. Interfaces that translate neural information from the brain into control commands, so called Brain Machine Interfaces (BMIs), are also studied for providing control over robotic devices [5]. Some of the aforementioned interfaces are also considered for space applications. However, additional requirements are posed, e.g. on weight and complexity. Furthermore, it is difficult to evaluate the devices in a real environment due to limited experimental time in mircogravity or even true space conditions. Nevertheless, a few approaches for teleoperation have been already tested in space environment.

For long distance (robotic) applications, as presented in [6], [7], [8], discrete command strategies and a high level of abstraction in combination with highly autonomous robot behavior is required due to the latency in the communication link. Graphical User Interfaces (GUI) provided on a computer screen or a tablet are used to trigger tasks or subtasks [9], [10].

Use cases with lower latency allow for direct control strategies based on continuous commands. One approach was investigated in the Kontur-2 mission in 2015-2017 [11], [12]. Here, a 2D force-feedback joystick was used on board the ISS to provide a cosmonaut with control over a robot on earth, while receiving haptic feedback of the external forces acting on the robot. Similarly, but extended to a 6-degree of freedom (DoF) force feedback device, an astronaut on the ISS used a sigma 7 device to control a rover as well as the equipped robotic manipulator during the Analog-1 mission of the European Space Agency [13]. 
All the aforementioned interfaces require an external input device, like a tablet or a joystick. To avoid this additional device, the use of BMIs for space applications is also discussed as an alternative [14]. Especially during EVAs the use of these biosignals can be useful, as the mobility is limited when wearing a space suit [15] and even may prevent the use of any external device at all.

Although non-invasive BMIs are seen as potential interfaces for space applications, no BMI has yet been used in a space mission. Current devices face inherent limitations like the amount of information content in the signals [16], or they can often not fulfill needed characteristics for space applications regarding their size or usability [15]. Furthermore, according to [15], BMIs for space applications should preferably be non-invasive and provide high reliability and efficiency while offering sufficient comfort to the operator with a low weight and volume. Additionally, [16] suggests for BMIs to be wireless and with a small number of sensors, when intended for space use.

An alternative that fits the aforementioned requirements for a wearable interface is the use of electromyography (EMG). The measured biosignals can be used to realize an interface between the user and a remote controlled device like a robot arm [17]. In previous work [18], we have realized an interface based on electromyography, which enables the operator to generate control signals for teleoperation through a defined activation of the muscles. Wearable sensors which are placed on the surface of the skin (sEMG) are used to record muscle activation, from which a continuous $3-\mathrm{DoF}$ output signal is generated to teleoperate a robotic device. Applying forces with the hands against an rigid body in different directions leads to reproducible variations in the EMG-signal. These biosignals can be processed with little delay, such that an online approach is possible. Basically, our interface based on EMG allows to resemble the functionality of a joystick but without the need of a stationary device. Up to now this input device has only been used in the health care sector to give people with severe motor impairments the possibility to remotely control devices like a tablet or a robot. In [19] the functionality of the interface could be demonstrated for people suffering from advanced muscular atrophy. These results suggest that the EMG-based interface can therefore be used if the user's muscle activity is altered or low, as may be the case with long-term stays in space. In our opinion, this interface meets most of the requirements highlighted for the use of BMIs in space applications, except for using a different kind of biosignal.

Sensorimotor performance and its impairment in weightlessness plays an important role when using the interface, as muscle contractions are used specifically for the realization of the interface. For the investigations of sensorimotor performance in weightlessness mainly aiming and tracking tasks are used, as they give insights about precise or continuous motor control abilities as response to visually sensed input. This is relevant to evaluate controlled movements but also to evaluate the usage of a motor controlled interface, which becomes more important with the increase of teleoperated systems in space missions.

From prior research it is known, that motor performance is affected by weightlessness [20]. Previous studies, like [21] or [22], investigated free arm movements in a tracking or aiming task and the resulting effects in weightlessness. The affected sensorimotor performance is often explained by distorted proprioception [23] or by additional cognitive load (during dual tasks) [22]. Altered control strategies, which are necessary to compensate for evoked counter forces, are also reported to cause slower limb motion [24], [25]. Furthermore, it has been proven that not only the body movement but also the force production is influenced by changed gravity conditions [26].

The effect on performance when controlling a joystick in weightlessness has also been investigated. Decreasing performance measures were recorded in different studies [27], [28]. In [28] the authors state that degraded proprioceptive feedback is one of the reasons for exaggerated force produced in a study simulating weightlessness by water immersion. Given that weightlessness can have a negative effect on motor performance, the question arises whether this influence affects the usage of an EMG-based interface in microgravity compared to usage on ground.

Studies on affected motor performance in weightlessness also investigate directional influences. While [29] could not find any directional differences in free arm movements, [30] found directional errors in the gravity axis (z-axis) in an aiming task. During joystick applications asymmetric influences of weightlessness on horizontal and vertical directions were recorded in [31], as well as in [32]. Following these findings, the second question arises whether the absence of gravity leads to directional influences on the EMG-based interface.

As the usage of the interface is based on applying forces with the hands against a rigid object like a handle, the body-pose stability and thus the motor performance could be affected when using the interface in microgravity without any further fixation of the body. In this case, the muscles of the arm are required to control the interface as well as to stabilize the body at the same time. It was found that trunk fixations are efficient to maintain performance during arm movements in weightlessness [22]. A missing fixation of the feet in microgravity can further influence the reference point of the body which is used for stabilization of the center of mass [33]. Therefore, a third research question arises how much user fixation is needed in order to operate via the interface.

The main contribution of this work is to investigate the aforementioned research questions: An exploratory user study should show whether negative influences of microgravity on operator performance using the EMG-based interface occur in general, to what extent they are direction-specific and whether a fixation of the operator has an influence. An aiming as well as a tracking task, as shown in [34] and [12], are performed from skilled operators in a parabolic flight study. Three different levels of fixation - fixed feet to the ground using foot straps, semi-free by using a foot rail, and freefloating feet - were examined to investigate the question of the needed operator fixation. 


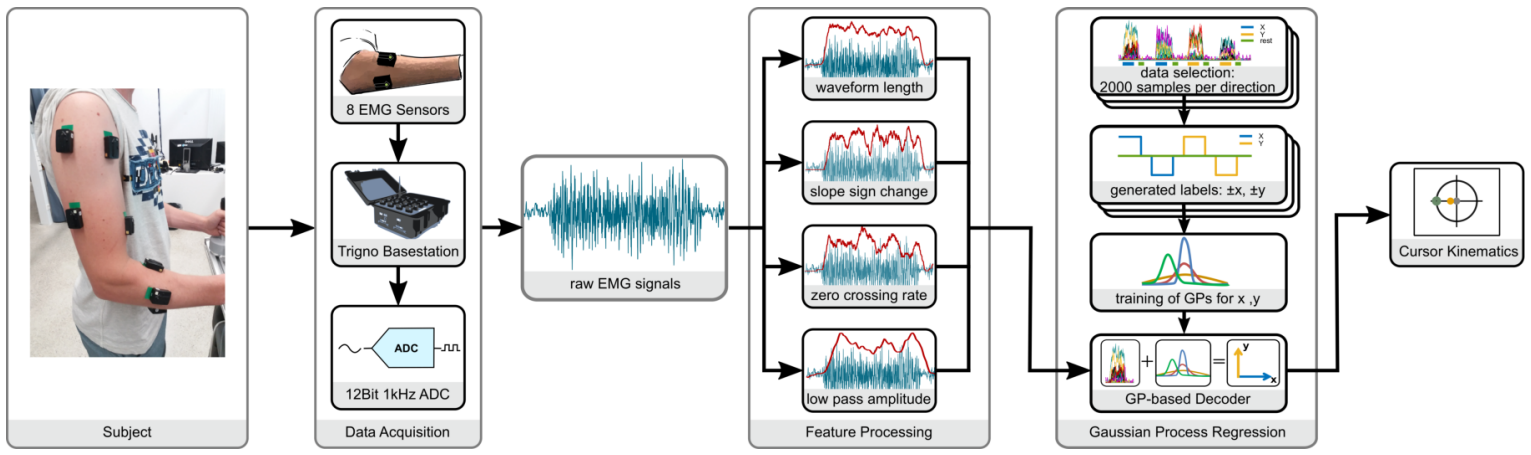

Fig. 2. Schematic overview of the signal processing pipeline; Subject: showing the electrode placement on the skin of the operator's arm; Data Acquisition: process of converting the sEMG-signals to digital signals; Feature Processing: using a sliding window of 150 samples to calculate the four time-domain features; Gaussian Process Regression: training procedure to get an GP-based decoder using three labeled training sets; Cursor Kinematics: the application to be controlled.

\section{METHODS}

\section{A. The sEMG-based Interface}

The used human machine interface is based on muscle contractions of the user, measured via sEMG and processed to create a command signal for a remote controlled system like a robot. The biosignals are mapped to a directional, velocitybased 2D (or 3D) control input. The schematic overview of the interface is given in Fig 1.

During this experiment, subjects were instructed to hold on to two handles in front of them and to apply forces and torques to be associated with the different directions of a 2D application (up, down, left and right). Having fixed handles allowed for a reproducible isometric muscle contraction without functional movement of the limbs. To realize the interface based on these isometric muscle activation, subjects were asked to produce forces and torques for the individual directions in a way they find intuitive and comfortable. This results in an interface, that is meant to be intuitive to use, as the subject is not required to learn specific muscle activation, but can freely decide how to map forces and torques to directional motion commands.

In this study eight wireless Delsys Trigno EMG-sensors were used to gather electromyographic data. The electrodes were distributed over the forearm and upper arm to cover the various muscle sites involved in force and torque production of the users' hand and arm. To record hand and wrist activity, electrodes were placed close to the muscles M. flexor digitorum superficialis, M. flexor carpi radialis, M. extensor carpi radialis, and $\mathrm{M}$. extensor digitorum, respectively. Two sensors were attached to the upper arm, i.e. the M. biceps brachii and to the M. triceps brachii, and two to the M. deltoid (anterior and posterior). The sensor placement is shown in Fig. 2.

Raw biosignals from the arm were recorded, amplified and wirelessly transferred to the Delsys Trigno Base Station. This receiver provided a $\pm 5 \mathrm{~V}$ analogue output signal, which was digitized on a $\mathrm{AD}$-converter of the company Beckhoff $(1 \mathrm{KHz}$, 12 bit) and transmitted to a real time computer via EtherCAT.

From the raw signals, a total of four different timedomain features were calculated: Waveform-Length, SlopeSign-Change, Zero-Crossing-Rate, and sEMG-amplitude. This set of features encodes important information for pattern recognition and was initially proposed by [35] to classify myoelectric patterns for the control of a multifunction prosthesis. The features were calculated on each of the eight EMGchannels using a sliding window of 150 samples. Finally, the mapping from the EMG-signals to the directional velocity commands was done using Gaussian Process Regression (see [18] for implementation details).

After applying the electrodes, initialization of the interface was necessary for each user. Therefore, the user had to complete a supervised calibration phase before starting. At first, the user was asked to hold on to the handles in a comfortable position without any additional muscular activity. For one, the sEMG-signal recorded in this resting state was used in order to determine and remove the signal's DC-offset. Additionally, a threshold-based parameter was calculated to distinguish the rest-signals from voluntarily activated muscle signals (activity detection). To obtain a baseline including variations, the resting data was recorded for five seconds.

Afterwards, training data from the user had to be recorded and labeled to generate the predictive model based on Gaussian Processes. Therefore, the subject was asked to produce forces and torques to be associated with motion along the cardinal axes $( \pm \mathrm{x}$ and $\pm \mathrm{y})$ in a predefined sequence. Subjects were instructed to produce muscular activity until at least 2000 samples ( 2 seconds) of activity, based on the output of the activity detection, were recorded. After each direction, the subject was required to relax for 1 second to improve separability of the labels within the training data. The whole procedure of one training cycle took between 14 and 18 seconds. In order to create variability in the EMG signals, four of these training cycles were recorded out of which the last three were used to train the Gaussian Processes. From each dataset and each direction, the first 2000 samples of directional activity and 1000 consecutive samples of rest were added to the training dataset (see Fig.2).

With this training data, two independent Gaussian Processes were used, one for the $\pm \mathrm{x}$ direction (left and right) and one to the \pm y direction (up and down). Having individual models also allowed the user to create a combination of the directions, resulting in diagonal motion commands. Additionally, to allow the subject to stop moving and hold the position of the controlled device, motion commands of the Gaussian Processes are only applied if the activity detection is positive, 
whereas motion commands are set to zero otherwise. Once the predictive Gaussian models were calculated, which took about 1 minute on the computers used in this experiment, the mapping from the EMG-input to the control output could be executed online. The hardware and software structure had a maximum delay smaller than $100 \mathrm{~ms}$, with a command rate of $100 \mathrm{~Hz}$, which allowed an online application without notable lag. Fig. 2 gives an overview of the signal processing pipeline.

\section{B. Subjects}

Six right handed subjects (three men and three women) in the age between 25 and 37 and a body height between $1,57 \mathrm{~m}$ and $1,83 \mathrm{~m}$ took part in the experiments. None of them had known neurological diseases or other physical impairments. Two of them had no parabolic flight experience before. The other four had less than three previous flights, none of them being part of a comparable experiment. All subjects were checked for airworthiness by an aviation physician prior to signing up for the campaign. The health status of each volunteer was checked again by a physician at the first day of the flight-week. In the morning of each flight, the subjects were injected with a standard dose of Scopolamine $(0.5 \mathrm{mg}$ for female and $0.7 \mathrm{mg}$ for male subjects) against motion sickness. Some subjects reported a slight dizziness for about 30 minutes after the injection, which disappeared before the experiments started. There were no reported abnormalities regarding dizziness or nausea during the flights. Ethical approval of the experimental procedure was given by the Comite de Protection des Personnes Sud-Ouest et Outre-mer III (ID RCB number: 2019-A00020-57). All subjects gave written consent to the procedure, which was explained to them orally and in written form.

\section{Experimental Tasks and Conditions}

In this study the EMG-based interface enabled the continuous velocity-based control of a cursor in 2D on a screen. Here, two different tasks were performed by the subjects: an aiming task and a tracking task. These types of tasks are often used for sensorimotor investigations, as they allow for analysis of directional gross and fine motion and quality of control at constant velocity.

A 14 inch display with a resolution of $1920 \mathrm{x} 1080 \mathrm{px}$ was used in front of the subjects to visualize the tasks as shown in Fig. 3. Crosshairs in $x-y$ directions were visualized with thin black lines. The cursor which was controlled by the subjects was depicted as a filled circle. During the Aiming Task (AT), a target was shown as a non-filled circle in green located in one of the four directions along the coordinate axis (cf. Fig. 3-AT). The starting point was always set to the center of the screen, and the subjects had to stay in rest (without movement of the cursor) for 2 seconds before a trial was started. The target to reach was located $400 \mathrm{px}$ up, down, left or right of the starting point. Subjects were instructed to reach the respective target and stay within the target area for $500 \mathrm{~ms}$ to finish the task successfully. They were asked to complete the task as fast as possible, since the duration of the tasks was used for the analysis. After a target was reached, the next trial started
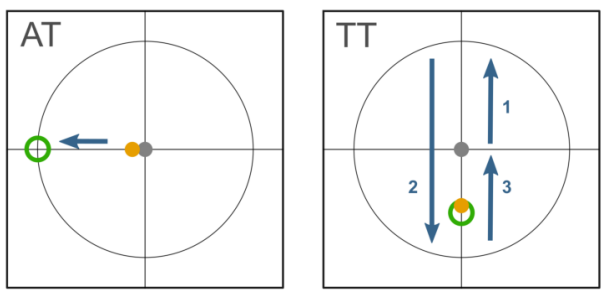

Fig. 3. Task description of the Aiming Task (left) and the Tracking Task (right). The yellow dot displays the cursor controlled by the subjects. The grey dot displays the starting point in the middle of the coordinate axis. The green circle shows the respective target. Whereas during AT one target was displayed along the cardinal axis which had to be reached as fast as possible, the target moved with constant velocity in horizontal or vertical direction during the TT.

and the target was displayed at a new randomly ordered target located in another direction along the cardinal axis. One test sequence included all four possible directions of the target. Each sequence was performed five times, resulting in a total of 20 trials per subject. The implementation of the AT is based on the experiments conducted by [34].

During the Tracking Task (TT) the target-circle moved either along the horizontal or the vertical axis, while the subjects should stay inside the target as precisely as possible. The cursor as well as the target-circle was initially placed at the same location in the center of the screen. The movement started always either towards the right or upwards until the target reached the outward limit, followed by a motion to the opposite side and finally back to the center (cf. Fig.3-TT). In total the distance which was covered by the target was $1600 \mathrm{px}$ within a duration of 16 seconds either in vertical or horizontal direction. The upcoming direction was visualized to the subjects before the task started. One test sequence of TT included the movement of the target in vertical and horizontal direction, which was repeated five times. The implementation of the TT is based on the experiments executed in [12].

To obtain comprehensive results about the influence of microgravity on operator performance in dependency of the fixation, three different test conditions were examined. The conditions refer to different fixation levels of the subjects' feet during microgravity (shown in Fig. 4). These conditions are:

- fixed, where the feet of the subjects were fixed to the ground using adjustable foot straps; the subjects could stabilize their body through their fixated feet as well as through the hands, which they held on to the handles

- semi-free, in which a foot rail replaced the foot straps; the feet were partially free so that small movements of the feet in all directions were possible; stabilization by hands was still given

- free, the feet of the subjects were not fixed at all; only stabilization through the hands was possible

Since all subjects had little to no experience with parabolic flights, the order of the conditions had to be the same for all subjects: 1) fixed, 2) semi-free, 3) free. Counterbalancing was not possible as the order was mandatory due to safety requirements of the parabolic flight. 


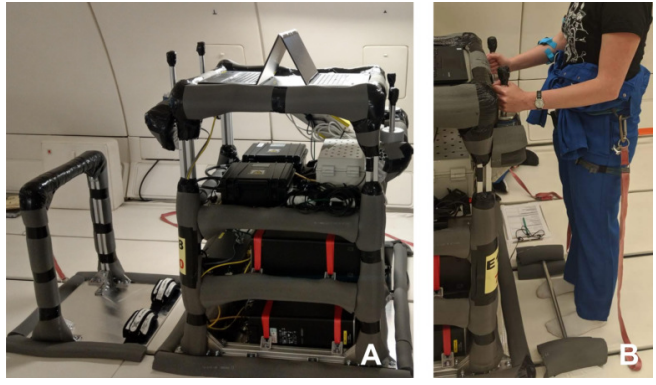

Fig. 4. Inflight setup with different fixations; The main rack included all equipment needed. The structure was mirrored so that two subjects could perform their tasks simultaneously; A: fixed condition including foot straps and a seating; B: subject in position during a semi-free session; the setup included a bar on the ground. The bar was removed for the free condition.

\section{Experimental Setup}

The setup, which is shown in Fig. 4, was built in an experimental rack, in compliance with the required aircraft safety regulations. In order to test two subjects simultaneously during each flight, the setup included two test stations, one on each side of the rack. Each test station included two vertically mounted handles, which were placed at the rack such that the subjects could hold on to them with angled elbows. The height and distance between the handles were adjusted individually to each subject's comfort. The display to visualize the tasks for the subjects was mounted on top of the rack. As described in II-A, eight EMG-electrodes were attached to the subject's dominant arm (here, always the right arm), as shown in Fig. 2.

Each of the three different conditions, which were introduced in II-C, required individual adaptations to the experimental setup. In the first condition adjustable foot straps were installed, as depicted in Fig. 4-A. Within the second condition a foot rail replaced the foot straps (Fig.4-B). This rail was modeled after the example of the rails that are mounted in the ISS [36]. A distance of nearly $4 \mathrm{~cm}$ between the ground and the foot rail enabled the semi-free test series. In the free test series, no fixation for the feet was present at all.

For safety reasons, a seating post was installed behind the subjects during the first flight which allowed the subjects to rest between the trials and especially during hyper-G phases. However, this seating post was removed during semi-free and free, but here, an additional harness attached to the subjects' waist should prevent uncontrolled floating.

\section{E. Experimental Procedure}

The experiments took part in the 33rd and 34th DLR parabolic flight campaign at NOVESPACE, in Bordeaux, France. The two flight campaigns were separated in time by six months. The flights were conducted in an A310 at the Mérignac International Airport in Bordeaux. All experiments of condition one, which include the fixation of the feet to the ground, were conducted in the 33rd DLR campaign by all six subjects. The experiments of semi and fully free feet took part in the 34th DLR campaign and were conducted by four subjects. All tests, including pre- and posttests were performed either in the plane (on ground) or at an identically constructed setup in the premises of NOVESPACE.
As the number of trials per subject is limited, due to the parabolic flight procedure, all subjects were trained and instructed in detail prior to the flights. This training procedure included a one-hour training once a week for a period of six weeks before the first campaign and a one-hour refreshing training for four weeks prior to the second parabolic flight campaign. In addition to the knowledge about the test procedure, the pre-training should ensure that all subjects have the same amount of training regarding the EMG-based interface, especially as all of them had prior knowledge at EMG-based interfaces but on different levels. During each training session, the subjects had to perform the AT and the TT, including two calibration phases and two phases of free-practice, where the subjects were asked to try out the different directional commands for 3 minutes.

To compare the performance between microgravity and the terrestrial conditions of $1 \mathrm{G}$, pre- and posttests were conducted one day before, and one day after the flight, respectively. In order to take training or fatigue effects into account, the same duration and trial-rhythm as in the inflight experiments have been implemented in the pre- and posttests. The specific placement of the electrodes was marked with medical grade permanent marker during the pretests to guarantee the identical electrode placement for the entire test series.

Each parabolic flight took about $3.5 \mathrm{~h}$ and contained 31 parabolas. The flight sequence followed the typical flight scheme hereafter: each parabola started and ended with a hypergravity phase of $1.8 \mathrm{~g}$ for about 24 seconds, and 22 seconds, respectively. In between the microgravity phase took place, which lasted 22 seconds. The pause between individual parabolas was about 1 minute 45 seconds, during which the subjects could rest. Longer breaks of 5 and 8 minutes were made after a block of 5 parabolas. Subjects only performed their tasks during microgravity. No measurements were conducted in regular- $\mathrm{G}$ and hyper- $\mathrm{G}$ phases. The first parabola, named $\sharp 0$, was used for familiarization of the subjects to microgravity without any test procedure being performed. In parabola $\sharp 1$ the rest signal of the right arm was measured as a basis of muscular activity in microgravity. In parabola $\sharp 2-5$ the subjects repeated four times the training procedure (once per parabola) of producing muscular activity associated with motion along the cardinal axis $( \pm \mathrm{x}$ and $\pm \mathrm{y}$ ). As the calibration phase of the system could be realized in a short time, it was possible to calibrate the system also during conditions of $0 \mathrm{G}$, within a total of five parabolas. The test trials were conducted from parabola $\sharp 6$. Due to the limited time in weightlessness (22 seconds), the duration of the experimental tasks had to be adjusted. During the AT, the maximum time to reach a target was limited to 8 seconds. The trial duration of the TT was adjusted to 16 seconds, in order to perform one trial within one parabola. All tasks started automatically after the onset of weightlessness. An acceleration sensor mounted on the setup monitored gravity at all times. Once microgravity set in $(0 \mathrm{G} \pm 0.3)$, the task was started; this way, the limited time of weightlessness could be used optimally. An additional process checked (during the AT) at any time if a further trial with a maximum of 8 seconds would fit completely within the ongoing parabola. Only if this condition was fulfilled the next 


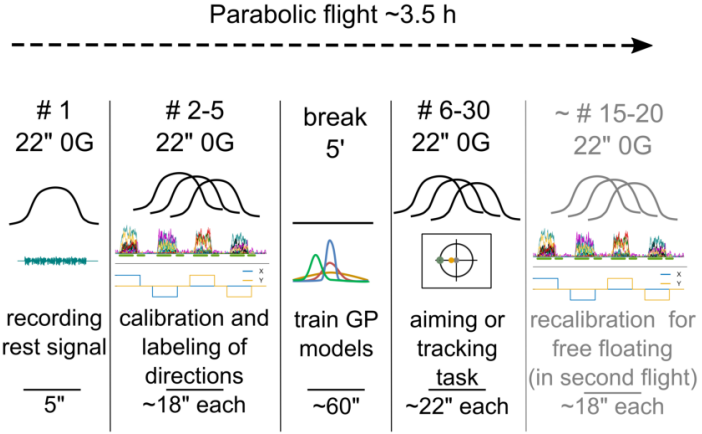

Fig. 5. Inflight procedure: the first parabola was used to record the muscular activity in the resting state; in parabola $\sharp 2-5$ initialization and data labeling took place; within the first break the model was trained; during the second campaign a second calibration phase was conducted for experiments with free-floating feet. All other parabolas were used to perform the tasks.

trial was started.

The calibration and the prediction models were acquired individually for each experimental condition. During fixed, subjects performed the AT, followed by the TT. As the semifree and free condition was tested within one flight, only the AT could be conducted. The subjects always started with the semi-free condition, followed by the free experiment. Each test (AT and TT) consists of one test trial at the beginning, followed by five full repetitions of the actual test. The whole procedure is illustrated in Fig. 5.

\section{F. Performance Measurement and Data Analysis}

From the collected data, four main performance measures were derived regarding the AT. These include the success rate, the reaction time, gross motion time, and fine motion time. The reaction time is defined as the time until the cursor was moved by at least 20 pixels. The gross motion time describes the time from the start of a trial until the cursor touches the target for the first time. The fine motion corresponds to the time after this first contact until the cursor is positioned correctly in the target zone and remains inside for $500 \mathrm{~ms}$. After finishing the fine motion, the trial was completed successfully. If a trial could not be completed within 8 seconds, the trial counted as failure. Not touching the target-circle at all was counted as gross motion failure. In total 120 trials per session were performed over all subjects during the tests of fixed feet and 80 during the other conditions (due to four instead of six subjects). To evaluate the TT, the average distance from the cursor to the target was calculated per trial.

Several statistical analyzes were conducted using a two-way repeated measures ANOVA. AT: The factors Gravity (pretest, inflight test, and posttest) and Direction (up, down, left, right) were used to analyze the performance measures gross motion, fine motion and reaction time for all three levels of fixations (nine tests). Further analyses have been performed on the results of the inflight tests with the factors Fixation (fixed, semi-free, and free) and Direction (three tests). TT: The factors Gravity (pretest, inflight test, and posttest) and Direction (horizontal and vertical) were used to analyze the average distance of the semi-free test series (one test).
In case of non-sphericity, Greenhouse-Geisser correction was conducted. For post hoc analysis a paired t-test with Bonferroni corrections was made. Failed trials, when not reaching the target within the time limit of 8 seconds, are not considered in this statistical analysis as no values are available from those trials.

\section{RESULTS}

The first condition of fixed was conducted by all six subjects (S1-S6). The test series involving the semi-free and free condition were performed by S1-S4. Therefore, the analysis of the factor Fixation is only based on the four subjects that completed all conditions. The Appendix contains detailed results of the performance measures in Table I, results of the statistical tests in Table II, as well as Fig. 10 which illustrates results of the AT achieved during all conducted test sessions within this study in a chronological order.

\section{A. Performance in microgravity vs. ground conditions}

1) Fixed: The subjects were able to successfully complete $89 \%$ (107 out of 120) of the Aiming Task during the parabolic flight. The ground tests show a success rate of about $96 \%$ (230 out of 240). In most of the failed trials fine motion could not be finished: i.e. ten times during the inflight test and nine times during the ground tests.

An analysis of the performance measures shows variations within the inflight test. Fig. 6 (left) illustrates the results for this test series. It is evident that the fine motion time increases during the flight. However, a significant main effect could not be revealed by the ANOVA $(\mathrm{F}(2,10)=3.68$, n.s. $)$. The small increase of gross motion time showed also no significant effect between the different gravity conditions. The reaction time shows a constant performance over the three sessions.

The results of the TT for the pre-, post- and inflight tests are illustrated in Fig. 7. The average distance from the cursor to the target shows increased mean values as well as a stronger deviation during the inflight test compared to the ground tests. The conducted ANOVA on the within factors Gravity and Direction could not reveal any significant main effects. Also, the interaction with the factors showed no effect.

Differences in the performance measures can be observed when considering each subject individually, as shown in Fig. 8. While the mean distance from cursor to target is clearly increased in weightlessness for S5, no negative influence can be observed during the inflight test for subjects S1, S2, S4, and S6. S3 shows a tendency to slightly better tracking results during microgravity.

2) semi-free: The mean values of the fine motion time achieved in microgravity show comparable values to those generated on ground (cf. Fig. 6 - middle). The gross motion time shows a slight increase of mean values and deviation only within the posttest, not within the inflight test. The average reaction time appears to be stable over the three sessions. In total $96 \%$ success rate could be reached in all sessions, independent of gravity. A significant main effect regarding the within factors Gravity and Direction could not be found by the repeated measures ANOVA. 


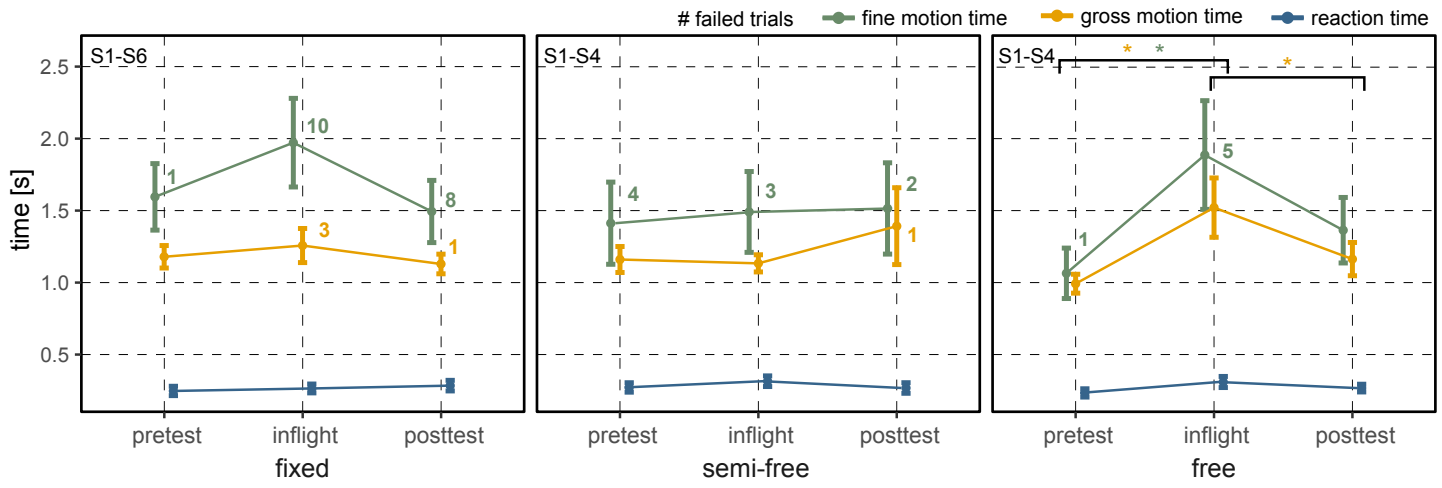

Fig. 6. Mean values of gross motion, fine motion, and reaction time of all test series with different fixations, composed of fixed, semi-free, and free. The numbers next to the graphs show the failed trials, i.e. trials which could not be finished within 8 seconds. The test series of fixed was performed by six subjects S1-S6; the other two test series were performed by S1-S4.

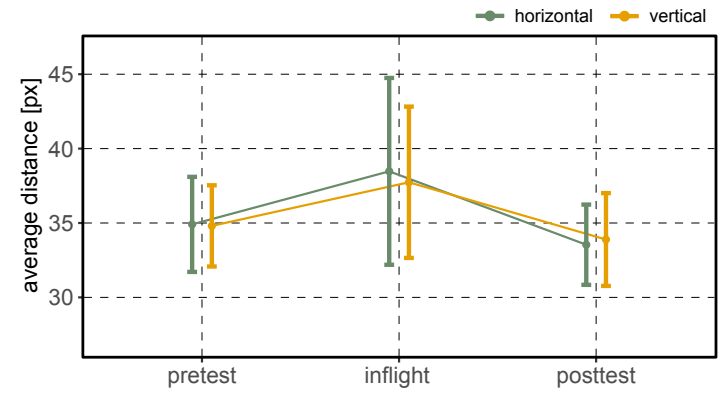

Fig. 7. Average distance from the controlled cursor to the target point during the Tracking Task (TT). The maximum distance covered by the target was $1600 \mathrm{px}$. The two different task-directions, including vertical and horizontal, are shown. The subjects S1-S6 during pretest, inflight test, and posttest are shown. The test was conducted within the condition fixed.

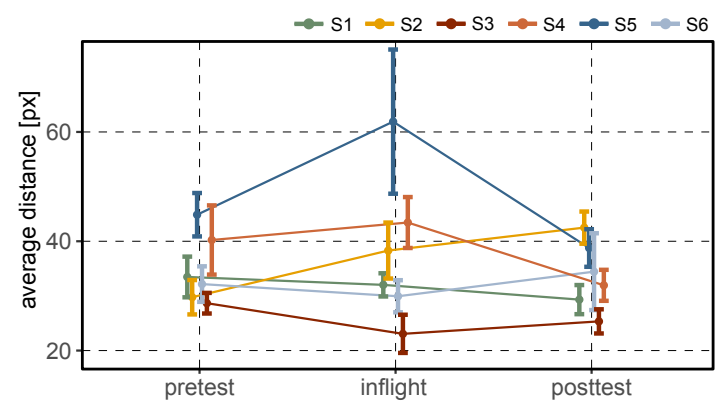

Fig. 8. Average distance from the controlled cursor to the target point per subject and test during the Tracking Task. The subjects S1-S6 during pretest, inflight test, and posttest are shown. The test was conducted within the condition fixed.

3) free: An effect of the microgravity compared to $1 \mathrm{G}$ could be revealed in the test series with free-floating feet. The ANOVA showed a significant main effect of the within factor Gravity in two cases: fine motion time $(\mathrm{F}(2,6)=5.68$, $\mathrm{p}<.05)$ and gross motion time $(\mathrm{F}(2,6)=6.35, \mathrm{p}<.05)$. The post hoc test indicated a significant difference between the pre- and the inflight test regarding the fine motion time. For the gross motion time the significant difference is given from both ground tests compared to the inflight test. A minimum of the completion time was reached within the pretest of free, which was conducted (equivalent to the inflight test) directly after the

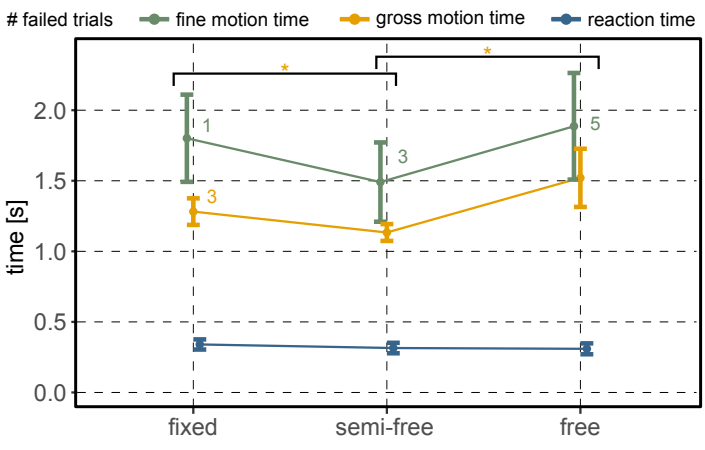

Fig. 9. Mean values of gross motion, fine motion, and reaction time of the three different conditions of the inflight experiment. Included are the subjects S1-S4, which performed all conditions. The numbers next to the graph show the failed trials.

pretest of semi-free. Five trials could not be completed within the flight and one trial in one of the pretests. Additionally, it has to be mentioned, that 15 of the inflight trials could not be performed by the subjects due to the limited time available in microgravity. A significant effect of the reaction time could not be found. These results are illustrated in Fig. 6 on the right side.

\section{B. Directional influence}

The statistical evaluation showed that the factor Direction has no significant influence on the performance. The twoway repeated measures ANOVAs could not identify any effect either of the factor itself or the interaction with this factor. Details of the analysis can be found in Table II in the Appendix.

\section{Performance in different levels of fixation}

Comparing the inflight tests with each other, a significant influence of the different types of fixations can be noticed within the gross motion time $(\mathrm{F}(2,6)=7.51, \mathrm{p}<.05)$. The post hoc test indicated a significant difference between semifree and the other two conditions fixed and free. A trend of a decreased completion time is visible during the semi-free condition for gross and fine motion time. The reaction time shows no influence of the different conditions. Fig. 9 compares the performance measures of all inflight tests of S1-S4. 


\section{DISCUSSION}

In this study, the effect of microgravity on task performance while using an interface based on electromyography in parabolic flights was investigated. To obtain comprehensive results, three different fixations of the feet during the flights have been applied. Pre- and posttests on the ground have been conducted in the same time scheme as the inflight tests to receive comparable results between ground $(1 \mathrm{G})$ and inflight test $(0 \mathrm{G})$ performance.

An influence of microgravity on the subjects' performance could only be identified to a small extent. The average success rate of more than $90 \%$ during the inflight tests confirm this small effect of the changed conditions. The success rates of the inflight tests in comparison to the ground tests are partially decreased, while there exists no difference for the semi-free condition (fixed: 0G: 89\%, 1G: $96 \%$; semi-free: 0G: $96 \%, 1 \mathrm{G}: 96 \%$; free: 0G: $92 \%, 1 \mathrm{G}: 99 \%$ ). In the analysis of reaction, gross and fine motion time, a measurable effect between $0 \mathrm{G}$ und $1 \mathrm{G}$ could not be detected within the test series of fixed and semi-free. Only the test series with free-floating feet revealed significant differences for the comparison between $0 \mathrm{G}$ and $1 \mathrm{G}$. The effect was revealed in analyzing gross and fine motion time, which both showed worse results during the flight tests compared to the ground tests (cf. Table I).

Both performance measures, gross and fine motion, show fluctuations during the study, with the fine motion time showing comparably broader variation (cf. Table I). This is partly to be expected, as haptic feedback plays a comparably larger role in fine motion than in gross motion [31]. The EMG-based interface used in this experiment provides haptic feedback, however, this is related to the input signals generated by the user when pushing on the handles. As this input is nonlinearly mapped to velocity commands, using Gaussian Process Regression, this haptic feedback does not necessarily correlate to the cursor's movement.

The analysis proved that there is no influence to the performance of the subjects regarding the direction of the task. When using an EMG-based interface, isometric muscle activity is used, a movement is not needed here. How and which muscle groups encode the different directions is decided individually by the user. These individual strategies can compensate for weaker directions and allow for equal directional control. Experiments with ISS astronauts using a joystick for teleoperation report asymmetric influences of the weightlessness on the different directions [31]. That experiment was performed similarly by using a second handle and foot rails in order to guarantee stabilization of the astronaut. The way the muscles are used during the control of the two interfaces (isometric forces vs. deflection of the limb) represents an essential difference of the EMG-based interface and a joystick. We hypothesize that isometric muscle activation may not be as sensitive to directional effects as non-isometric movements.

Sensorimotor performance is, as reported in literature ([20], [21], [23]), affected in weightlessness. In our study, the performance measures decreased during $0 \mathrm{G}$ within the test series of fixed. This is evident in the aiming task as well as in the tracking task. However, we could not identify a substantial effect across all subjects in this study. A previous study showed further that there exists a negative effect of microgravity on humans capability to produce defined forces [27]. This would suggest, that a degradation of performance could also emerge in our experiment. However, the calibration of the EMG-based interface was done under the same gravity condition as the tasks. Therefore, the muscle forces used for the respective commands originated from the same condition, which may have compensated for this effect.

The results of semi-free show no difference of performance during weightlessness. Gross and fine motion time had stable results in all tests, irrespective of the gravity. We hypothesize that these differences can be attributed to a learning effect regarding posture and body awareness under microgravity. All subjects started with the condition fixed within the first parabolic flight. The second flight included semi-free as well as free. This order was identical for all subjects due to safety reasons. As a result, all subjects had already experience with using the EMG-based interface in a parabolic flight environment, as well as with the inflight test procedure when performing the semi-free test series. Learning and adapting to effects on the proprioception or occurring counter forces, may have been achieved to a large part during the first flight, thereby improving subjects' performance during semi-free.

Improved performance during the semi-free condition also suggests, that the change from fully fixed feet to the foot rail had no strong influence to the body-pose stability of the subjects. For body stabilization the center of mass plays a decisive role. The reference point for stabilization of the center of mass remains also during weightlessness at the feet, as long as the feet are fixed to the ground [33]. In our experiment, the foot rail seems to provide sufficient means of support to maintain the feet as reference, as the constant performance suggests that no further disturbances to the sensorimotor system have been evoked under this condition.

The absence of a feet fixation causes a shift from a groundbased reference point to a head-based reference point [33]. As a result, reorganization of body posture and movement is necessary. We assume, that these effects partly cause the decreased performance during free. Additionally, in the freefloating condition, pushing and pulling on the handles, in order to evoke the correct EMG-activity, will create forces which affect the body posture. Furthermore, it is known that movements in weightlessness can lead to counter forces in other parts of the body, an effect which is typically compensated by altered control strategies [24], [25]. However, adaptive processes of sensorimotor organization occur during long-term space flights [24], which could not be considered in this study.

In our experiment, subjects had to quickly learn how to deal with these effects, as time in this condition was very limited (at max. 15 parabolas in free). However, the high success rates of about $92 \%$ show that the subjects still had control over the operated cursor. The tasks could be processed and completed in most cases. A long-term investigation in a free-floating condition would be needed to show, if this performance would settle to $1 \mathrm{G}$ levels once the operator is used to the conditions.

The fact, that the reaction time remains nearly constant, 
suggest that subjects are not influenced by higher cognitive load during the tasks in weightlessness. The authors in [31] found that in joystick applications during weightlessness feedforward motions are primarily affected by higher cognitive load, whereas feedback driven movements are influenced by the evoked proprioceptive disorder. Feedback dependent motions are described as most parts of the gross as well as the whole fine motion, whereas the feedforward motion is described by the reaction time and the initial part of the gross motion. Our results with affected gross and fine motion and constant reaction time are in line with the results of [31].

Considering the individual performance of the TT as shown in Fig. 8, differences can be recognized between the subjects. From literature it is known, that there exist discrepancies how people react to weightlessness [23]. As all subjects had no profound experience in weightlessness the individual influence of this factor was not predictable. A further test series on the ISS could give evidence how strong these effects act on a skilled astronaut.

Conducting the experiment within a parabolic flight campaign posed considerable demands on the interface and the subjects. However, the efficient calibration routine and intuitive handling of the interface allowed to perform the experiment given these constraints. In addition, the lightweight design of the interface did not affect the subjects' mobility and therefore we assume that no additional side effects result from the interface itself. This is supported by the fact that subjects were able to execute at the same performance during the flight as on the ground. The aforementioned characteristics of the EMG-based interface are an advantage over other interfaces based on biosignals. For example, a mobile application using systems such as magnetoencephalography (MEG) or functional magnetic resonance imaging (fMRI) would not be possible at present due to the size of the system. An online application as shown in this study, could also pose a problem to BMIs based on near infrared spectroscopy (NIRS) or fMRI as the underlying biological process, the haemodynamic response, has a delay of 2-5 seconds [16]. Other interfaces like electroencephalography (EEG) still require long and intense calibration routines, which limits their use in practice [37], especially with regard to the time constraints of a parabolic flight. Methods based on eye-tracking may also be suitable input devices to be considered as mobile interface for space applications. However, such systems do not allow the user to observe the environment as the line of sight is directly linked to task execution. Additionally, control in 3D is still a main challenge in the field of gaze tracking [38].

Given the aforementioned challenges and the results of our study, the usage of muscle signals seems to be a promising option, for uni-modal wearable interfaces based on biosignals. While mobility of the interface is an advantage over a conventional and typically stationary joystick, precision will need to be improved in order to use this interface in an actual space application. An additional limitation of the EMG-based interface is the already mentioned missing haptic feedback as well as the need of a rigid object (like handles) to evoke the needed muscle activation. In robotic applications, missing feedback and precision may be compensated by means of shared- or supervised-control strategies. Additionally, the EMG-based interface could potentially be adapted to a handsfree approach which could be useful for the usage in space suits during EVAs.

\section{CONCLUSION}

This study indicates that weightlessness affects the usage of an interface based on electromyography only to a small extent. The subjects which had very little experience with being in weightlessness, could handle the changed conditions such that the generated muscle signals are stable and reproducible enough to use the interface during weightlessness. Directional influences on operator performance were not detected in this study. A measurable effect of microgravity is not evident in two of the three test series. Both kinds of fixation of the feet allowed the subjects to perform the tasks in $0 \mathrm{G}$ without significant difference to $1 \mathrm{G}$ conditions. Significant degradation of performance is only noticeable in experiments with free floating feet, still, a high success rate is to be observed.

We assume that foot straps or a foot rail provide a similar degree of posture awareness and stabilization, as the results improved during the second inflight test series when using the foot rail. Hence a full fixation of the feet is not needed for a successful control of the interface. Rails as those already installed inside the ISS, would allow the usage of the EMGbased interface with similar performance as on the ground. A mobile application in the space station should be feasible as such rails and handles are mounted all over the ISS. As we assume an adaptation to microgravity, it could be hypothesized, that performance even in free floating condition may converge to that in $1 \mathrm{G}$ state, once users have more experience in weightlessness. The authors advocate further experiments with astronauts using the interface in weightlessness to provide more insights about the performance of a person used to this condition. A positive outcome would endorse the usage of the interface during EVAs as well.

This first investigation indicates a high potential for the use of EMG-based interfaces for teleoperation in space. Matching the requirements posed in [15], [16] our interface is portable and small in size, and provides an efficient calibration routine, which can easily be performed in weightlessness. The system allows a direct, continuous control in 2D as well as an indirect control (selection and navigation between tasks on a screen), which covers a wide range of applications. The extension to a 3D application should be feasible, as this was already in use in a previous terrestrial application [19].

Existing limitations may be compensated by approaches of shared and supervised control strategies, when teleoperating a robotic device. Current developments on EMG-based interfaces, like an individual sensor placement for each user, could further improve the performance of the interface for later applications. Experiments with astronauts on the ISS would be the next step to take this application forward. 


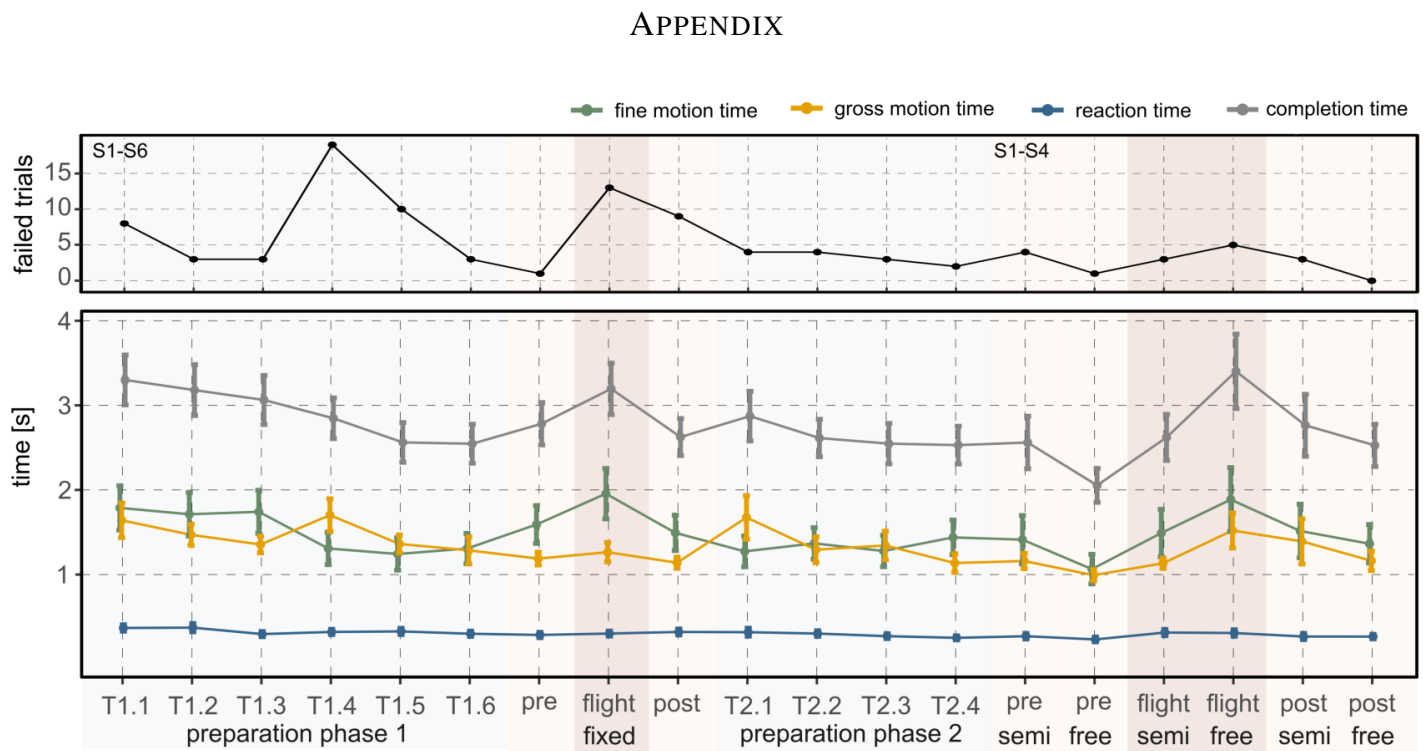

Fig. 10. Mean values of the performance measures of all conducted AT-tests of this study including the two preparation phases prior to the main experiments. The first preparation phase started six weeks prior to the experiments containing a one-hour training each week. The second preparation training contains four identical sessions and started about six months after the posttest of the condition fixed. The preparation phase 1, the main experiment of fixed, and preparation phase 2 was conducted by six subjects; The main experiment of semi-free (here called semi) and free (here called free) was conducted by four subjects. The upper part of the figure shows the failed trials of each session.

TABLE I

RESUlts of AIMING AND TRACKING TASKS OF ALL FIXATIONS AND OF ALL PERFORMANCE MEASURES INCLUDING GROSS MOTION, FINE MOTION, AND REACTION TIME AS WELl AS AVERAGE DISTANCE AND COMPLETED TRIALS. CONSIDERED ARE S1-S6 FOR fixed, AND S1-S4 FOR semi-free AND free.

\begin{tabular}{|c|c|c|c|c|c|c|c|}
\hline \multirow{2}{*}{ Fixation } & \multirow{2}{*}{ Measure } & \multicolumn{2}{|c|}{ Pretest } & \multicolumn{2}{c|}{ Inflight test } & \multicolumn{2}{c|}{ Posttest } \\
\cline { 3 - 8 } & & mean \pm sd & completed & mean \pm sd & completed & mean \pm sd & completed \\
\hline \multirow{4}{*}{ Fixed } & gross motion time [s] & $1.19 \pm 0.41$ & $120 / 120$ & $1.23 \pm 0.45$ & $117 / 120$ & $1.13 \pm 0.36$ & $119 / 120$ \\
\cline { 2 - 8 } & fine motion time [s] & $1.59 \pm 1.23$ & $119 / 120$ & $1.95 \pm 1.55$ & $107 / 117$ & $1.49 \pm 1.11$ & $111 / 119$ \\
\cline { 2 - 8 } & reaction time [s] & $0.28 \pm 0.17$ & $120 / 120$ & $0.31 \pm 0.16$ & $120 / 120$ & $0.31 \pm 0.17$ & $120 / 120$ \\
\cline { 2 - 8 } & avg.distance [px] & $34.86 \pm 7.89$ & $60 / 60$ & $38.10 \pm 15.17$ & $60 / 60$ & $33.71 \pm 7.74$ & $60 / 60$ \\
\hline \multirow{3}{*}{ Semi-Free } & gross motion time [s] & $1.16 \pm 0.40$ & $80 / 80$ & $1.13 \pm 0.27$ & $80 / 80$ & $1.25 \pm 0.78$ & $79 / 80$ \\
\cline { 2 - 8 } & fine motion time [s] & $1.41 \pm 1.24$ & $76 / 80$ & $1.49 \pm 1.23$ & $77 / 80$ & $1.51 \pm 1.40$ & $77 / 79$ \\
\cline { 2 - 8 } & reaction time [s] & $0.27 \pm 0.14$ & $80 / 80$ & $0.31 \pm 0.16$ & $80 / 80$ & $0.26 \pm 0.16$ & $80 / 80$ \\
\hline \multirow{3}{*}{ Free } & gross motion time [s] & $0.99 \pm 0.29$ & $80 / 80$ & $1.52 \pm 0.79$ & $65 / 65$ & $1.16 \pm 0.52$ & $80 / 80$ \\
\cline { 2 - 8 } & fine motion time [s] & $1.06 \pm 0.77$ & $79 / 80$ & $1.88 \pm 1.46$ & $60 / 65$ & $1.36 \pm 1.022$ & $80 / 80$ \\
\cline { 2 - 8 } & reaction time [s] & $0.23 \pm 0.12$ & $80 / 80$ & $0.31 \pm 0.16$ & $65 / 65$ & $0.27 \pm 0.12$ & $80 / 80$ \\
\hline
\end{tabular}

TABLE II

RESULTS OF THE TWO-WAY REPEATED MEASURES ANOVA; FACTORS FOR AT: GRAVITY (PRETEST, INFLIGHT TEST, AND POSTTEST), DIRECTION (UP, DOWN, LEFT, AND RIGHT), AND FIXATION (FIXED, SEMI-FREE, AND FREE); FACTORS FOR TT: GRAVITY AND DIRECTION (HORIZONTAL AND VERTICAL).

\begin{tabular}{|c|c|c|c|c|c|}
\hline \multirow{2}{*}{ Measure } & \multicolumn{5}{|c|}{ Two-way repeated measures ANOVA } \\
\hline & \multicolumn{3}{|c|}{ AT: Gravity(G)*Direction(D) } & $\begin{array}{c}\text { AT: Fixation(Fix)*Direction(D) } \\
\text { S1-S4 }\end{array}$ & $\begin{array}{c}\text { TT: Gravity(G)*Direction(D) } \\
\text { S1-S6 }\end{array}$ \\
\hline \multirow{2}{*}{$\begin{array}{l}\text { gross motion } \\
\text { time }[\mathrm{s}]\end{array}$} & $\mathrm{G}: \mathrm{F}(2,10)=2.88$ & $\mathrm{G}: \mathrm{F}(2,6)=0.77$ & G: $F(2,6)=6.35, p<.05$ & Fix: $F(2,6)=7.51, p<.05$ & \multirow[t]{2}{*}{$\mathrm{Cr}_{\mathrm{C}}$} \\
\hline & $G^{*} D: F(6,30)=0.51$ & $\mathrm{G}^{*} \mathrm{D}: \mathrm{F}(6,18)=0.89$ & $G^{*} D: F(6,18)=0.62$ & Fix $* D: F(6,18)=0.81$ & \\
\hline \multirow{3}{*}{$\begin{array}{l}\text { fine motion } \\
\text { time }[\mathrm{s}]\end{array}$} & $\mathrm{G}: \mathrm{F}(2,10)=3.68$ & G: $F(2,6)=0.11$ & G: $F(2,6)=5.68, p<.05$ & Fix: $F(2,6)=0.91$ & \multirow{3}{*}{-} \\
\hline & D: $F(3,15)=2.97$ & $D: F(3,9)=0.91$ & D: $F(3,9)=0.10$ & D: $F(3,9)=0.07$ & \\
\hline & $\mathrm{G}^{*} \mathrm{D}: \mathrm{F}(6,30)=0.44$ & $\mathrm{G}^{*} \mathrm{D}: \mathrm{F}(6,18)=0.82$ & $G^{*} D: F(6,18)=0.38$ & Fix*D: $F(6,18)=0.21$ & \\
\hline $\begin{array}{l}\text { reaction } \\
\text { time }[\mathrm{s}]\end{array}$ & $G^{*} D: F(6,30)=0.65$ & $G^{*} D: F(6,18)=0.71$ & $G^{*} \mathrm{D}: \mathrm{F}(6,18)=3.95$ & Fix $* D: F(6,18)=1.35$ & - \\
\hline \multirow{3}{*}{$\begin{array}{c}\text { average } \\
\text { distance }[\mathrm{px}]\end{array}$} & \multirow{3}{*}{ - } & \multirow{3}{*}{ - } & \multirow{3}{*}{-} & \multirow{3}{*}{ - } & $\mathrm{G}: \mathrm{F}(2,10)=0.75$ \\
\hline & & & & & D: $F(1,5)=0.04$ \\
\hline & & & & & $G^{*} \mathrm{D}: \mathrm{F}(2,10)=0.18$ \\
\hline
\end{tabular}




\section{ACKNOWLEDGMENT}

The authors would like to thank the DLR Space Administration as well as NOVESPACE, which supported the experiments within the 33rd and 34th DLR Parabolic flight campaign. We thank Bernhard Weber for his valuable input to this manuscript and all subjects of the study. B. Eskofier gratefully acknowledges the support of the German Research Foundation (DFG) within the framework of the Heisenberg professorship programme (grant number ES 434/8-1).

\section{REFERENCES}

[1] International Space Exploration Coordination Group, "The global exploration roadmap," p. https://www.globalspaceexploration.org/, January 2018.

[2] R. Ambrose, B. Wilcox, B. Reed, L. Matthies, D. Lavery, and D. Korsmeyer, "Robotics, tele-robotics and autonomous systems roadmap: Technology area 04," National Aeronautics and Space Administration (NASA), 2012.

[3] A. Tobergte, P. Helmer, U. Hagn, P. Rouiller, S. Thielmann, S. Grange, A. Albu-Schäffer, F. Conti, and G. Hirzinger, "The sigma. 7 haptic interface for mirosurge: A new bi-manual surgical console," in 2011 IEEE/RSJ International Conference on Intelligent Robots and Systems. IEEE, 2011, pp. 3023-3030.

[4] M. Sagardia, K. Hertkorn, T. Hulin, S. Schätzle, R. Wolff, J. Hummel, J. Dodiya, and A. Gerndt, "VR-OOS: The DLR's virtual reality simulator for telerobotic on-orbit servicing with haptic feedback," in IEEE Aerospace Conference. IEEE, 2015, pp. 1-17.

[5] L. F. Nicolas-Alonso and J. Gomez-Gil, "Brain computer interfaces, a review," Sensors, vol. 12, no. 2, pp. 1211-1279, 2012.

[6] A. Schiele, "Meteron-validating orbit-to-ground telerobotics operations technologies," in 11th Symposium on Advanced Space Technologies for Robotics and Automation (ASTRA), 2011.

[7] E. Dupuis, P. Langlois, J. L. Bedwani, D. Gingras, A. Salerno, P. Allard, S. Gemme, R. L'Archevêque, and T. Lamarche, "The avatar explore experiments: results and lessons learned," in Proceedings of the International Symposium on Artificial Intelligence, Robotics, and Automation in Space, 2010.

[8] A. K. Tanwani and S. Calinon, "A generative model for intention recognition and manipulation assistance in teleoperation," in 2017 IEEE/RSJ International Conference on Intelligent Robots and Systems (IROS). IEEE, 2017, pp. 43-50.

[9] M. Diftler, T. Ahlstrom, R. Ambrose, N. Radford, C. Joyce, N. De La Pena, A. Parsons, and A. Noblitt, "Robonaut 2-initial activities on-board the ISS," in 2012 IEEE Aerospace Conference. IEEE, 2012, pp. 1-12.

[10] P. Schmaus, D. Leidner, T. Krüger, R. Bayer, B. Pleintinger, A. Schiele, and N. Y. Lii, "Knowledge driven orbit-to-ground teleoperation of a robot coworker," IEEE Robotics and Automation Letters, vol. 5, no. 1, pp. 143-150, 2019.

[11] J. Artigas, R. Balachandran, C. Riecke, M. Stelzer, B. Weber, J.-H. Ryu, and A. Albu-Schaeffer, "Kontur-2: force-feedback teleoperation from the international space station," in 2016 IEEE International Conference on Robotics and Automation (ICRA). IEEE, 2016, pp. 1166-1173.

[12] B. Weber, S. Schätzle, C. Riecke, B. Brunner, S. Tarassenko, J. Artigas, R. Balachandran, and A. Albu-Schäffer, "Weight and weightlessness effects on sensorimotor performance during manual tracking," in International Conference on Human Haptic Sensing and Touch Enabled Computer Applications. Springer, 2016, pp. 111-121.

[13] M. Panzirsch, H. Singh, T. Krüger, C. Ott, and A. Albu-Schäffer, "Safe interactions and kinesthetic feedback in high performance earth-to-moon teleoperation," IEEE Aerospace Conference, 2020.

[14] L. Rossini, D. Izzo, and L. Summerer, "Brain-machine interfaces for space applications," in 2009 Annual International Conference of the IEEE Engineering in Medicine and Biology Society. IEEE, 2009, pp. 520-523.

[15] C. Menon, C. De Negueruela, J. d. R. Millán, O. Tonet, F. Carpi, M. Broschart, P. Ferrez, A. Buttfield, F. Tecchio, F. Sepulveda et al., "Prospects of brain-machine interfaces for space system control," Acta Astronautica, vol. 64, no. 4, pp. 448-456, 2009.

[16] E. B. Coffey, A.-M. Brouwer, E. S. Wilschut, and J. B. van Erp, "Brainmachine interfaces in space: using spontaneous rather than intentionally generated brain signals," Acta Astronautica, vol. 67, no. 1-2, pp. 1-11, 2010.
[17] P. K. Artemiadis and K. J. Kyriakopoulos, "EMG-based control of a robot arm using low-dimensional embeddings," IEEE Transactions on Robotics, vol. 26, no. 2, pp. 393-398, 2010.

[18] J. Vogel and A. Hagengruber, "An sEMG-based interface to give people with severe muscular atrophy control over assistive devices," in 2018 40th Annual International Conference of the IEEE Engineering in Medicine and Biology Society (EMBC). IEEE, 2018, pp. 2136-2141.

[19] A. Hagengruber and J. Vogel, "Functional tasks performed by people with severe muscular atrophy using an sEMG controlled robotic manipulator," in 2018 40th Annual International Conference of the IEEE Engineering in Medicine and Biology Society (EMBC). IEEE, 2018, pp. $1713-1718$

[20] J. R. Lackner and P. DiZio, "Human orientation and movement control in weightless and artificial gravity environments," Experimental brain research, vol. 130, no. 1, pp. 2-26, 2000.

[21] O. Bock, S. Abeele, and U. Eversheim, "Sensorimotor performance and computational demand during short-term exposure to microgravity," Aviation, space, and environmental medicine, vol. 74, no. 12, pp. 12561262, 2003.

[22] B. Fowler, S. Meehan, and A. Singhal, "Perceptual-motor performance and associated kinematics in space," Human factors, vol. 50, no. 6, pp. 879-892, 2008

[23] O. Bock, "Problems of sensorimotor coordination in weightlessness," Brain research reviews, vol. 28, no. 1-2, pp. 155-160, 1998.

[24] M. Berger, S. Mescheriakov, E. Molokanova, S. Lechner-Steinleitner, N. Seguer, and I. Kozlovskaya, "Pointing arm movements in short-and long-term spaceflights." Aviation, space, and environmental medicine, vol. 68, no. 9, pp. 781-787, 1997.

[25] S. Mechtcheriakov, M. Berger, E. Molokanova, G. Holzmueller, W. Wirtenberger, S. Lechner-Steinleitner, C. De Col, I. Kozlovskaya, and F. Gerstenbrand, "Slowing of human arm movements during weightlessness: the role of vision," European journal of applied physiology, vol. 87, no. 6, pp. 576-583, 2002.

[26] A. Mierau and M. Girgenrath, "Exaggerated force production in altered Gz-levels during parabolic flight: The role of computational resources allocation," Ergonomics, vol. 53, no. 2, pp. 278-285, 2010.

[27] A. Mierau, M. Girgenrath, and O. Bock, "Isometric force production during changed-Gz episodes of parabolic flight," European journal of applied physiology, vol. 102, no. 3, pp. 313-318, 2008.

[28] M. Dalecki, T. Dräger, A. Mierau, and O. Bock, "Production of finely graded forces in humans: effects of simulated weightlessness by water immersion," Experimental brain research, vol. 218, no. 1, pp. 41-47, 2012.

[29] J. Fisk, J. R. Lackner, and P. DiZio, "Gravitoinertial force level influences arm movement control," Journal of neurophysiology, vol. 69 , no. 2, pp. 504-511, 1993.

[30] H. E. Ross, "Motor skills under varied gravitoinertial force in parabolic flight," Acta Astronautica, vol. 23, pp. 85-95, 1991.

[31] B. Weber, C. Riecke, and M. Stelzer, "Disentangling the enigmatic slowing effect of microgravity on sensorimotor performance," in Proceedings of the Human Factors and Ergonomics Society Europe Chapter 2019 Annual Conference. HFES-Europe, 2019.

[32] B. Weber, M. Panzirsch, F. Stulp, and S. Schneider, "Sensorimotor performance and haptic support in simulated weightlessness," Experimental Brain Research, vol. 238, no. 10, pp. 2373-2384, 2020.

[33] J. Massion, B. Amblard, C. Assaiante, L. Mouchnino, and S. Vernazza, "Body orientation and control of coordinated movements in microgravity," Brain Research Reviews, vol. 28, no. 1-2, pp. 83-91, 1998.

[34] B. Weber, S. Schätzle, and C. Riecke, "Comparing the effects of space flight and water immersion on sensorimotor performance," in Proceedings of the Human Factors and Ergonomics Society Europe Chapter 2017 Annual Conference. HFES online, 2017.

[35] B. Hudgins, P. Parker, and R. N. Scott, "A new strategy for multifunction myoelectric control," IEEE Transactions on Biomedical Engineering, vol. 40, no. 1, pp. 82-94, 1993.

[36] NASA, "Human integration design handbook (HIDH)," National Aeronautics and Space Administration (NASA), p. https://www.nasa.gov/, June 2014.

[37] F. Lotte, "Signal processing approaches to minimize or suppress calibration time in oscillatory activity-based brain-computer interfaces," Proceedings of the IEEE, vol. 103, no. 6, pp. 871-890, 2015.

[38] C. Elmadjian, P. Shukla, A. D. Tula, and C. H. Morimoto, "3d gaze estimation in the scene volume with a head-mounted eye tracker," in Proceedings of the Workshop on Communication by Gaze Interaction, 2018, pp. 1-9. 